\title{
artigo
}

\section{Como melhorar a qualidade da saúde da equipe de enfermagem mediante ao COVID-19}

\author{
How to improve the quality of health of the nursing staff through COVID-19
}

Cómo mejorar la calidad de salud del equipo de enfermería ante el COVID-19

\begin{abstract}
RESUMO
Objetivo: Identificar estratégias que podem ser inseridas ou já utilizadas, para promover a saúde dos trabalhadores de enfermagem no enfrentamento do Sars Cov 2 e melhorar a rotina de trabalho. Métodos: Este estudo se afigura como uma revisão integrativa de literatura, este trabalho versa sobre artigos em língua portuguesa, espanhol e inglês, do período de março de 2020 a outubro de 2020. Os critérios de inclusão foram: Texto completo de acesso gratuito, ensaio clínico, meta-Análise, ensaio controlado randomizado e revisão sistemática. Resultados: Foram encontrados ao todo 133 produtos nas bases de dados PubMed, Lilacs e SciELO. Sendo estes 3 duplicados e após a leitura foi deduzido que 120 destes trabalhos não preenchiam os critérios estabelecidos. Conclusão: Foi possível perceber com este estudo, que a maioria dos fatores que afetam a saúde do trabalhador de enfermagem, está diretamente relacionado às questões psíquicas, que devem ser trabalhadas com investimento de apoio psiquiátrico e psicológico frequentemente. A inclusão de tecnologias que mantém o profissional distante a alguns casos, se mostra satisfatório
\end{abstract}

DESCRITORES: Enfermeiros. Pandemia. Covid-19. Saúde ocupacional.

\section{ABSTRACT}

Objective: To identify strategies that can be inserted or already used, to promote the health of nursing workers in coping with Sars Cov 2 and improve the work routine. Methods: This study appears as an integrative literature review, this work deals with articles in Portuguese, Spanish and English, from March 2020 to October 2020. The inclusion criteria were: Full text of free access, essay clinical, meta-analysis, randomized controlled trial and systematic review. Results: A total of 133 products were found in the PubMed, Lilacs and SciELO databases. These 3 being duplicated and after reading it was deduced that 120 of these works did not meet the established criteria. Conclusion: It was possible to perceive with this study, that most of the factors that affect the health of the nursing worker, are directly related to psychic issues, which must be worked with investment of psychiatric and psychological support frequently. The inclusion of technologies that keep the professional away from some cases is satisfactory

DESCRIPTORS: Nurses. Pandemic. Covid-19. Occupational health.

\section{RESUMEN}

Objetivo: Identificar estrategias que puedan ser insertadas o ya utilizadas, para promover la salud de los trabajadores de enfermería en el afrontamiento del Sars Cov 2 y mejorar la rutina laboral. Métodos: Este estudio aparece como una revisión integrativa de la literatura, este trabajo trata con artículos en portugués, español e inglés, desde marzo de 2020 hasta octubre de 2020. Los criterios de inclusión fueron: texto completo de libre acceso, ensayo clínico, metaanálisis, aleatorizado controlado ensayo y revisión sistemática. Resultados: Se encontraron un total de 133 productos en las bases de datos PubMed, Lilacs y SciELO. Estos 3 están duplicados y tras su lectura se dedujo que 120 de estos trabajos no cumplían con los criterios establecidos. Conclusión: Se pudo percibir con este estudio, que la mayoría de los factores que inciden en la salud del trabajador de enfermería, están directamente relacionados con problemas psíquicos, los cuales deben ser trabajados con inversión de apoyo psiquiátrico y psicológico con frecuencia. La inclusión de tecnologías que mantienen al profesional alejado de algunos casos es satisfactoria

DESCRIPTORES: Enfermeras. Pandemia. COVID-19. Salud Ocupacional.

RECEBIDO EM: 25/01/2021 APROVADO EM: 03/02/2021

\section{Joyce Lemos de Souza Botelho}

Enfermeira. Faculdades Santo Agostinho - Montes Claros MG (2020).

ORCID: 0000-0002-2284-9844 


\author{
Solange Macedo Santos \\ Enfermeira. Enfermeira. Faculdades Santo Agostinho - Montes Claros MG (2020). \\ ORCID: 0000-0002-1956-4880 \\ Carolaine Fiuza Aquino \\ Acadêmica de Enfermagem. Faculdades Santo Agostinho - Montes Claros - MG. \\ ORCID: 0000-0003-3127-1279 \\ João Marcos Oliveira de Melo \\ Acadêmico de Enfermagem. Faculdades Santo Agostinho - Montes Claros - MG. \\ ORCID: 0000-0002-4197-5504
}

Ingred Gimenes Cassimiro de Freitas

Enfermeira, Mestranda em Cuidado Primário. Supervisora de Estágio do curso de Enfermagem.Faculdades Santo Agostinho Montes Claros - MG.

ORCID: 0000-0001-8325-0985

\section{INTRODUÇÃO}

$\mathbf{N}$ o ano de 2019, no mês de novembro surgiu um novo coronavírus causando uma grande pandemia por uma infecção respiratória, esse vírus foi determinado como Sars-Cov2 $2^{1}$, que foi apresentado como um dos maiores desafios sanitários em escala global deste século ${ }^{2,3}$. Foi considerado Emergência de Saúde Pública de Importância Internacional, a partir da segunda reunião do comitê de emergência realizado na China em 30 de janeiro de 2020, pelo grande número de casos confirmados da doençá ${ }^{4}$. Foi sugerido pela Organização Mundial da Saúde (OMS) planos de contingência semelhante ao da influenza, pelas características sintomáticas respiratórias, no entanto com ações diferentes dependendo da gravidade da pandemia ${ }^{5}$. No Brasil foram notificados 6.628 .065 milhões casos confirmados ${ }^{6}$ por isso houve a necessidade do aumento da quantidade de leitos para COVID-19 segregados e aumento dos cuidados recomendados.

A demanda extra gerada pela pandemia aumenta a necessidade de oferta dos serviços de saúde, e é uma situação preocupante para aqueles serviços e municípios que não estão preparados para mudanças tão drásticas, sendo necessárias algumas medidas como hospitais de campanha ou leitos extras e alocação de profissionais de saúde para trabalhar de modo que não comprometa a saúde dos pacientes ${ }^{7}$. No início de maio o Brasil se encontrava em primeiro lugar no ranking de óbitos e possuía ainda uma grande quantidade de afastamentos seja por COVID-19 ou outros motivos ${ }^{8,9,10}$. Esses achados faz refletir sobre quais são os motivos para esses fatores, podendo ser o período de treinamento curto, ou má capacitação da instituição, jornada extensas, condições de trabalho ruins até mesmo o vínculo da vida pessoal e profissional deve ser avaliada ${ }^{11}$, pois são todos fatores importantes que interferem diretamente e indiretamente na saúde da equipe.

A saúde do trabalhador está no campo da saúde coletiva, tendo como foco o processo de saúde-doença e rotina laboral, buscando a transformação dos processos produtivos promovendo a saúde ${ }^{12}$. Além disso, tem se falado muito da equipe de enfermagem e médicos da linha de frente que estão extremamente expostos aos pacientes de quadros leves e graves, aumentando a carga viral recebida, somado ao mau uso dos equipamentos de proteção individual ou condições inadequadas de trabalho que causa grande nível de estresse psicológico e cansaço físico ${ }^{8}$. Todos esses fatores podem causar ao trabalhador um esgotamento profissional, caracterizado como uma síndrome geral de adaptação onde diversos mecanismos são desencadeados no organismo por causas desfavoráveis do ambiente levando ao quadro da síndrome de Burnout ${ }^{9}$.

Mediante a uma pandemia que exige ações de urgência, os gestores responsáveis pela equipe de saúde deve promover técnicas e ferramentas para melhorar a condição de trabalho precária ou difícil, que podem ser até mesmo sem custos, a fim de trazer melhores resultados para os serviços, bom atendimento para os pacientes e uma qualidade vida e trabalho melhor para o funcionário. $\mathrm{O}$ serviço de saúde em meio a uma pandemia se mostra crucial, sendo assim, as estratégias do cuidado integral entre os profissionais para combater a precarização do cuidado em saúde se tornam imprescindíveis para o avanço da enfermagem ${ }^{11}$.

Identificar estratégias que podem ser inseridas ou já utilizadas, para promover a saúde dos trabalhadores de enfermagem e minimizar danos causados pela pandemia.

\section{MÉTODOS}

O estudo classifica-se como uma revisão integrativa da literatura. A pesquisa foi pautada em 6 etapas: 1) Escolha do questionamento norteador; 2) Delimitação de critérios para inclusão; 3) Apresentação dos artigos; 4) Análise dos achados; 5) Interpretação de resultados obtidos; 6) Descrição das evidências. Tendo como questão norteadora: "Quais ferramentas e atitudes estão sendo tomadas para que a saúde do trabalhador da Enfermagem seja preservada mediante a pandemia da Sars Cov2?". $\mathrm{O}$ processo de busca dos artigos aconteceu entre os meses de Setembro a Dezembro de 2020. O levantamento foi realizado nas 


\section{artigo}

bases de dados elegíveis: Literatura Latino-Americana e do Caribe em Ciências da Saúde (LILACS), U.S. National Institute of Health (PubMed) e Scientific Electronic Library Online (SciELO).

Foram utilizados os operadores booleanos AND e OR, e as estratégias de busca foram: professional burnout OR hospital nursing service AND pandemics OR personal protective equipment $\mathrm{e}$ nas bases demais: equipe de enfermagem OR serviços de enfermagem AND pandemias OR esgotamento profissional, sendo validados por dois pesquisadores. Os critérios de inclusão inseridos neste trabalho foram a) Texto completo de acesso gratuito b) Ensaio Clínico, Meta-Análise, Ensaio Controlado Randomizado, Revisão Sistemática c) Do período de março a dezembro de 2020 d) Em inglês, portugues e espanhol. Foram realizadas análises descritivas, comparativas, onde foi possível descrever e comparar resultados obtidos em cada estudo, tornando viável a união desses artigos para uma melhor interpretação em cima do tema trabalhado.

\section{RESULTADOS}

Foram encontrados ao todo 133 artigos nas bases de dados PubMed, Lilacs e Scielo, sendo 3 duplicados e após a leitura 120 estudos não preenchiam os critérios de inclusão estabelecidos.

A amostra final foi constituída por 10 artigos que puderam ser selecionados mediante os critérios de classificação. No quadro 1 consta as especificidades trabalhadas em cada artigo. Desta forma foram observados escassos os resultados obtidos no que se refere ao cuidado com o profissional da enfermagem mediante a uma pandemia.

\section{DISCUSSÃO}

Em um momento de pandemia, os serviços de saúde procuram se adequar ao que tem até obter materiais necessários e como os profissionais de saúde não podem parar seus serviços dão continuidade aos cuidados. No entanto, estudo realizado em $2020^{13}$ evidencia ser de baixa exatidão que máscaras cirúrgicas ofereçam de forma semelhante a N95 a proteção contra as infecçôes de vias respiratórias, exceto em procedimento geradores de aerossóis, orientando até a preservação dos respiradores N95 para casos de escassez ou procedimentos supracitados $^{13}$. Apesar de não apresentar altas taxas de certeza, traz para a equipe e os gestores possibilidades junto com o conhecimento científico de diminuir o rigor do uso constante desse equipamento. A pandemia dos Sars Cov2 mostra um impacto relevante e acentuado na saúde física e também na mental dos profissionais da saúde, tornando-se uma prioridade para os serviços de saúde buscarem estratégias para contornar a situação $0^{14}$.

De acordo com 13 estudos incluídos na revisão integrativa de GARCÍA-IGLESIAS, 2020, diz que os profissionais de saúde da linha de frente estavam com as funções mentais comprometidas pela situação enfrentada, levando a altos níveis de stress, ansiedade, depressão, nervosismo e insônia. Ainda, podemos envolver problemas mentais após-surtos entre os

Quadro 1 - Artigos selecionados após processo de filtragem e seus respectivos resultados com alta relevância ao tema pressuposto.

\begin{tabular}{|c|c|c|c|c|}
\hline AUTOR & PAÍS/LOCAL & EPI'S & $\begin{array}{l}\text { SAÚDE MENTAL DOS } \\
\text { PROFISSIONAIS }\end{array}$ & SAÚDE FÍSICA DOS PROFISSIONAIS \\
\hline $\begin{array}{l}\text { Bartoszko et al., } \\
2020^{13}\end{array}$ & Canadá & $\begin{array}{l}\text { Coloca semelhança na pro- } \\
\text { teção do profissional entre a } \\
\text { máscara cirúrgica para a N95 }\end{array}$ & Não relatado & Proteção eficaz \\
\hline $\begin{array}{l}\text { Houghton et al., } \\
2020^{14}\end{array}$ & Inglaterra & $\begin{array}{l}\text { Difícil adesão visto com temor } \\
\text { pelos pacientes Desconforto }\end{array}$ & $\begin{array}{l}\text { Insegurança mediante } \\
\text { aos novos protocolos }\end{array}$ & $\begin{array}{l}\text { Em risco pelos fatores mentais e } \\
\text { ambientais }\end{array}$ \\
\hline $\begin{array}{l}\text { Chughtai et al., } \\
2020^{15}\end{array}$ & Paquistão & $\begin{array}{l}\text { Reaproveitamento uso } \\
\text { indevido }\end{array}$ & Não relatado & Exposição \\
\hline $\begin{array}{l}\text { Gonzalo et al., } \\
2020^{16}\end{array}$ & Espanha & Não relatado & Medo Insônia & Mialgia calafrios Fadiga \\
\hline $\begin{array}{l}\text { García-iglesias et } \\
\text { al., } 2020^{17}\end{array}$ & Espanha & Não Relatado & $\begin{array}{l}\text { Insônia Ansiedade } \\
\text { Nervosismo }\end{array}$ & Não Relatado \\
\hline Jiade et al., $2020^{18}$ & Boston & Dermatite ocupacional & Não relatado & Não relatado \\
\hline $\begin{array}{l}\text { Paiano et al., } \\
2020^{20}\end{array}$ & China & Insuficientes & Medo Estigma & Não relatado \\
\hline Wang et al., $2020^{21}$ & China & Doenças de pele & Não relatado & Não relatado \\
\hline $\begin{array}{l}\text { Carvalho et al., } \\
2020^{23}\end{array}$ & Brasil & Não relatado & Estresse & Exaustão \\
\hline Forte et al., $2020^{25}$ & Brasil & Falta de EPI's & Pressão psicológica & Cansaço \\
\hline
\end{tabular}


funcionários, levando a grande necessidade de apoio psicológico e psiquiátrico, levando a criação de estratégias a fim de amenizar tais danos, a partir da cultura distinta de cada país ${ }^{20}$. Trabalhadores da área da saúde possuem maior probabilidade de desenvolver estresse ocupacional, apesar de neste estudo haver uma baixa ocorrência de casos relatados ${ }^{23}$. O autor ressalta que esse fato pode ser devido já ter tido intervenção dos gestores, ou omissão por medo.

Mediante a uma situação de pandemia, em que toda a equipe está à frente de novas diretrizes e normas, as instituições devem garantir a segurança dos funcionários a partir do repasse de instruções à equipe, treinamentos para evitar o uso incorreto ou inapropriado, sendo a comunicação crucial para o atual momento ${ }^{18}$. Cabe então aos gestores a responsabilidade pelo êxito no repasse de informações necessárias à equipe, agindo de forma preventiva contra os danos causados por uma pandemia inesperada. Atuando também, de forma que a equipe não sofra pela falta de equipamentos, a exposição acontece fisicamente ao vírus e psicologicamente pela preocupação em contaminar seus amigos e familiares ${ }^{20}$. Os treinamentos para as equipes se tornam benéficas para que sejam incentivados a relatar formas erradas ou certas de atividades cometidas, a fim de compartilhar incidentes, para benefício do aprendizado com pessoas que lidam principalmente com riscos biológicos ${ }^{22}$.

Muitos pacientes necessitam de cuidados mesmo estando em isolamento, por isso, foi criado a telemedicina, onde o profissional pudesse atender e cuidar do doente sem sair do consultório, e sem o cliente também se expor externamente nas ruas. Pode-se utilizar tal estratégia a fim de orientar quanto ao uso das máscaras de proteção, limpeza doméstica, entre outros cuidados $^{21}$, minimizando a exposição também do profissional com o vírus.

Diversos projetos buscam desenvolver tecnologias rápidas e instantâneas, por exemplo, como atender e como deve ser as condutas de profissionais mediante a
Muitos pacientes

necessitam de

cuidados mesmo

estando em

isolamento, por

isso, foi criado a

telemedicina, onde o

profissional pudesse

atender e cuidar do

doente sem sair do

consultório, e sem

o cliente também se

expor externamente

nas ruas. Pode-se

utilizar tal estratégia

a fim de orientar

quanto ao uso

das máscaras de

proteção, limpeza

doméstica, entre

outros cuidados,

minimizando a

exposição também

do profissional

com o vírus. um paciente suspeito de COVID-19 dentro de hospitais, fortalecendo também o vínculo entre a área administrativa com a assistencialista, que acaba dependendo dessas tecnologias implantadas em sua rotina beneficiando a segurança dos profissionais ${ }^{24}$.

Para evitar o uso indevido de equipamentos de proteção individual (EPI), garantir a segurança dos profissionais de saúde e evitar a escassez, a comunicação eficaz de diretrizes de controle de infecção atualizadas é essencial. Como as equipes pré-hospitalares estão particularmente sob risco de contaminação devido ao seu ambiente de trabalho desafiador, um módulo específico de aprendizagem eletrônica gamificada (e-learning) direcionado a esse público pode fornecer vantagens significativas, pois não requer a presença de alunos nem o uso repetitivo de equipamentos para demonstração ${ }^{8}$.

Tornou-se notável a valorização da equipe de enfermagem e sua atuação no combate a pandemia, em diversas redes sociais pode-se encontrar manifestações e também agradecimentos. Mas também, serviu para que os trabalhadores pudessem reivindicar seus direitos pelas mídias sociais, obtendo um grande alcance em massa e tornando uma manifestação virtual por menos horas trabalhadas e mais equipamentos de proteção disponíveis ${ }^{25}$.

\section{CONCLUSÃO}

Foi possível perceber com este estudo, que a maioria dos fatores que afetam a saúde do trabalhador de enfermagem, está diretamente relacionada às questões psíquicas, que devem ser trabalhadas com investimento de apoio psiquiátrico e psicológico frequentemente. A inclusão de tecnologias que mantém o profissional distante a alguns casos, se mostra satisfatório, pois, a exposição exagerada e falta de equipamentos de proteção individual deixa-os mais preocupados com a própria saúde e dos familiares. O treinamento da equipe antes de se expor a uma pandemia, como atitude preventiva, passou mais confiança para a realização dos cuidados, 


\section{artigo}

o fato de algo ser imprevisto ou inesperado causou impactos em todos os profis- sionais. Além da valorização profissional faz-se necessário melhorar as condições de trabalho, para que eles possam cuidar dos pacientes, bem consigo mesmo.

\section{REFERÊNCIAS}

1. Coronavírus Brasil [Internet]. Saude.gov.br. 2021 [cited 2020 Set 29].

2. Folha informativa COVID-19 - Escritório da OPAS e da OMS no Brasil - OPAS/OMS | Organização Pan-Americana da Saúde [Internet]. Paho.org. 2021 [cited 2020 Out 20].

3. Xu Z, Shi L, Wang Y, Zhang J, Huang L, Zhang C, et al. Pathological findings of COVID-19 associated with acute respiratory distress syndrome. The Lancet Respiratory Medicine [Internet]. 2020 Apr [cited 2020 Out 20];8(4):420-2.

4. Novel Coronavirus [Internet]. Who.int. 2019 [cited 2020 out 20].

5. Freitas, André Ricardo Ribas, Napimoga, Marcelo e Donalisio, Maria Rita. Análise da gravidade da pandemia de Covid-19. Epidemiologia e Serviços de Saúde [online]. v. 29, n. 2 [Acessado 31 Outubro 2020].

6. Brasil. Ministério da Saúde. Painel de casos de doenças pelo coronavírus 2019 (COVID-19) no Brasil [Internet]. Brasília: Ministério da Saúde; 2020.

7. Noronha, Kenya Valeria Micaela de Souza et al. Pandemia por COVID-19 no Brasil: análise da demanda e da oferta de leitos hospitalares e equipamentos de ventilação assistida segundo diferentes cenários. Cadernos de Saúde Pública [online]. v. 36, n. 6, e00115320.

8. Teixeira, Carmen Fontes de Souza et al. A saúde dos profissionais de saúde no enfrentamento da pandemia de Covid-19. Ciência \& Saúde Coletiva [online]. v. 25, n. 9, pp. 3465-3474.

9. Vasconcelos EM de, Trindade CO, Barbosa LR, Martino MMF de. Predictive factors of burnout syndrome in nursing students at a public university. Revista da Escola de Enfermagem da USP [Internet]. 2020 [cited 2020 out 28];54.

10. Associação Brasileira de Saúde Coletiva [Internet]. Rio de Janeiro: ABRASCO; c2020 [citado 2020 out 31]. Machado MH. Profissionais de saúde em tempos de Covid-19; [aprox. 1 tela].

11. David HMSL, Acioli S, Silva MRF da, Bonetti OP, Passos H. Pandemics, crisis conjunctures, and professional practices: what is the role of nursing with regard to Covid-19? Revista Gaúcha de Enfermagem [Internet]. 2021 [cited 2020 Nov 19];42(spe).

12. Faria MG de A, Silveira EA da, Cabral GR da FC, Silva RO da, Daher DV, David HMSL. Saúde do trabalhador no contexto da estratégia de saúde da família: revisão integrativa de literatura. Escola Anna Nery [Internet]. 2020 [cited 2020 Nov 20];24(4).

13. BARTOSZKO, Jessica J et al. "Medical masks vs N95 respirators for preventing COVID-19 in healthcare workers: A systematic review and meta-analysis of randomized trials." Influenza and other respiratory viruses [Internet]. 2020 [cited 2020 Nov 21];14(4).

14. Houghton C, Meskell P, Delaney H, Smalle M, Glenton C, Booth $A$, et al. Barriers and facilitators to healthcare workers' adherence with infection prevention and control (IPC) guidelines for respiratory infectious diseases: a rapid qualitative evidence synthesis.
Cochrane Database of Systematic Reviews [Internet]. 2020 Apr 21 [cited 2020 Nov 22].

15. Chughtai $A A$, Khan W. Use of personal protective equipment to protect against respiratory infections in Pakistan: A systematic review. Journal of Infection and Public Health [Internet]. 2020 Mar [cited 2020 Nov 22];13(3):385-90.

16. Salazar de Pablo G, Vaquerizo-Serrano J, Catalan A, Arango C, Moreno C, Ferre F, et al. Impact of coronavirus syndromes on physical and mental health of health care workers: Systematic review and meta-analysis. Journal of Affective Disorders [Internet]. 2020 Oct [cited 2020 Nov 23];275:48-57.

17. García-Iglesias JJ, Gómez-Salgado J, Martín-Pereira J, Fagundo-Rivera J, Ayuso-Murillo D, Martínez-Riera, José Ramón, et al. Impacto del SARS-CoV-2 (Covid-19) en la salud mental de los profesionales sanitarios: una revisión sistemática. Ruauaes [Internet]. 2020 [cited 2020 Nov 23].

18. Yu J, Chen JK, Mowad CM, Reeder M, Hylwa S, Chisolm S, et al. Occupational Dermatitis to Facial Personal Protective Equipment in Healthcare Workers: A Systematic Review. Journal of the American Academy of Dermatology. 2020 Oct;

19. Suppan L, Abbas M, Stuby L, Cottet P, Larribau R, Golay E, et al. Effect of an E-Learning Module on Personal Protective Equipment Proficiency Among Prehospital Personnel: Web-Based Randomized Controlled Trial. Journal of Medical Internet Research [Internet].

20. Paiano $M$, Jaques $A E$, Nacamura $P A B$, Salci MA, Radovanovic CAT, Carreira L. Mental health of healthcare professionals in China during the new coronavirus pandemic: an integrative review. Revista Brasileira de Enfermagem [Internet]. 2020 [cited 2020 Nov 28];73(suppl 2).

21. Wang Y, Fang R, Zhang $H$, Tang K, Sun Q. Contributions of dermatologists to COVID 19 research: A brief systematic review. Dermatologic Therapy [Internet]. 2020 Jul [cited 2020 Dez 28];33(4).

22. Che Huei L, Ya-Wen L, Chiu Ming Y, Li Chen H, Jong Yi W, Ming Hung L. Occupational health and safety hazards faced by healthcare professionals in Taiwan: A systematic review of risk factors and control strategies. SAGE Open Medicine [Internet]. 2020 Jan [cited 2020 Dez 28];8:205031212091899.

23. Carvalho AEL de, Frazão I da S, Silva DMR da, Andrade MS, Vasconcelos SC, Aquino JM de. Stress of nursing professionals working in pre-hospital care. Revista Brasileira de Enfermagem [Internet]. 2020 [cited 2020 Dez 28];73(2).

24. Glauber F. Equipe Técnica Temporária Especializada em hospitais de pequeno porte: estratégia de controle do Covid-19. Enferm foco (Brasilia) [Internet]. 2020 [cited 2020 Dez 28];211-6.

25. Forte ECN, Pires DEP de. Nursing appeals on social media in times of coronavirus. Revista Brasileira de Enfermagem [Internet]. 2020 [cited 2021 Jan 28];73(suppl 2). 